\title{
1 Prediction of Whole-Cell Transcriptional Response with 2 Machine Learning
}

\begin{tabular}{|c|c|c|c|}
\hline Mohammed Eslami ${ }^{1, *,+}$ & Amin Espah Borujeni ${ }^{2,+}$ & Hamid Doosthosseini ${ }^{2}$ & Matthew Vaughn ${ }^{3}$ \\
\hline Hamed Eramian' ${ }^{1}$ & Katie Clowers $^{4}$ & D. Benjamin Gordon ${ }^{2}$ & Niall Gaffney ${ }^{3}$ \\
\hline Mark Weston $^{1}$ & Diveena Becker ${ }^{4}$ & Yuval Dorfan ${ }^{2}$ & John Fonner ${ }^{3}$ \\
\hline Joshua Urrutia $^{3}$ & Carolyn Corbet $^{4}$ & George Zheng $^{1}$ & Joe Stubbs ${ }^{3}$ \\
\hline \multirow[t]{2}{*}{ Alexander Cristofaro ${ }^{2,5}$} & Paul Maschhoff ${ }^{4}$ & Jedediah Singer $^{6}$ & \\
\hline & Christopher A Voigt ${ }^{2}$ & Enoch Yeung ${ }^{7, *}$ & \\
\hline
\end{tabular}

${ }^{1}$ Netrias, LLC, Annapolis, MD 21409, USA, ${ }^{2}$ Massachusetts Institute of Technology, Cambridge, MA 02139, USA, ${ }^{3}$ Texas Advanced Computing Center, Austin, TX 78758, USA, ${ }^{4}$ Ginkgo Bioworks, Inc., Boston, MA 02210, USA, ${ }^{5}$ TScan Therapeutics, Inc., Waltham, MA 02451, USA, ${ }^{6}$ Two Six Technologies, Arlington, VA 22203, USA, ${ }^{7}$ University of California Santa Barbara, Santa Barbara, CA 93106, USA

*To whom correspondence should be addressed. (meslami@netrias.com, eyeung@ucsb.edu )

${ }^{+}$Authors contributed equally. 


\section{Abstract}

6 Applications in synthetic and systems biology can benefit from measuring whole-cell response to

7 biochemical perturbations. Execution of experiments to cover all possible combinations of

8 perturbations is infeasible. In this paper, we present the host response model (HRM), a machine

9 learning approach that takes the cell response to single perturbations as the input and predicts the

10 whole cell transcriptional response to the combination of inducers. We find that the HRM is able

11 to qualitatively predict the directionality of dysregulation to a combination of inducers with an

12 accuracy of $>90 \%$ using data from single inducers. We further find that the use of known prior,

13 known cell regulatory networks doubles the predictive performance of the HRM (an $R^{2}$ from 0.3

14 to 0.65$)$. This tool will significantly reduce the number of high-throughput sequencing

15 experiments that need to be run to characterize the transcriptional impact of the combination of

16 perturbations on the host.

\section{Introduction}

18 Cells enact complex dynamics in response to environmental and biochemical perturbations. The

19 perturbation can have a widespread effect so as to alter the dynamics of the whole cell through

20 cascading effects that span through a cell's regulatory network. Combinations of the biochemical

21 perturbations are thus not additive and can trigger complex responses such as heat shock ${ }^{1,2}$,

22 osmotic shock ${ }^{3,4}$, or sudden shifts in nutrient availability ${ }^{5,6}$. Given the complexity of these

23 responses to these perturbations, prior studies in perturbed whole cell response examine the role

24 of a specific well-known biophysical perturbation, for which there is a natural intuition or 
25 sensible alignment with known biophysical mechanisms ${ }^{7,8,9}$. These experiments are carefully

26 performed, driven by biophysical knowledge and hypothesis-based modeling.

28 A natural extension of this research is to examine how the whole cell responds to inputs that are

29 foreign to the natural workings of the cell. Further, suppose that a cell was presented with

30 multiple inputs, each of which lent biophysical insight, but the goal was to predict how the cell

31 responded combinatorially to these inputs. The scale of experiments required to measure

32 response in such a combinatorially large condition space is infeasible in terms of cost, labor, and

33 time. In such a setting, there is great value in developing discovery-based approaches for

34 spotlighting biophysical mechanisms using data-driven algorithms ${ }^{10}$, such as nonlinear

35 modeling $^{11}$ or machine learning ${ }^{12}$.

37 A domain that has grappled with modeling of combinatorially large condition spaces for

38 prediction of specific cell response is that of drug combination/synergy prediction ${ }^{12,13}$. Machine

39 and deep learning techniques are widely used to model pharmacodynamic and pharmacokinetic

40 parameters of a drug and identify biomarkers of drug response given a large corpus of drug and

41 response features ${ }^{14-17}$. The techniques used in these efforts require large training datasets that

42 consist of specific cell responses to tens of thousands of drugs, a condition space that is often too

43 large for high-throughput omics measurements, such as RNASeq, which provide insight into the

44 whole-cell response. The ubiquity of high-throughput sequencing offers an opportunity to

45 revolutionize the modeling of whole cell transcriptional response. 
47 The ubiquity of high-throughput sequencing offers an opportunity to revolutionize the modeling

48 of whole cell transcriptional response. A measure most often used to qualitatively and

49 quantitatively assess a transcript's response is its dysregulation as compared to a control.

50 Differential expression analysis (DEA) is a standard bioinformatics technique that measures

51 response to perturbations as compared to a control condition ${ }^{18}$. DEA conducts custom

52 normalization, dispersion modeling, and Bayesian optimization to account for biological and

53 experimental variability in the data. It quantifies the transcriptional response to a perturbation in

54 terms of fold-change and measures its statistical significance. Data-driven prediction using

55 machine learning of transcription to date, however, has been limited to expression level

56 predictions from sequences or images ${ }^{19-20}$. These techniques are prone to generalization errors

57 that can arise from artifacts of normalization of counts data across experiments with

58 combinatorically large condition spaces $^{21}$.

60 In this paper we present the host response model (HRM), a machine learning model that can

61 predict whole-cell transcriptional response to a combination of biochemical perturbations using

62 transcriptional response data from single perturbations. Biochemical perturbations, in this

63 context, amounts to inducing a cell with a chemical. The HRM combines high-throughput

64 sequencing with machine learning to infer links between experimental context, prior knowledge

65 of cell regulatory networks, and the RNASeq data to predict differential expression of a gene.

66 The HRM was tested in two organisms, Escherichia coli MG1655 (E. coli) and Bacillus subtilis

67 Marburg 168 (B. subtilis). E. coli is a well-studied and characterized Gram-negative bacteria that

68 served as a proof of concept in the development of the HRM. B. subtilis is a well characterized 
69 and frequently used model organism for Gram-positive bacteria that was used to pressure test the

70 HRM. For conciseness, the figures for E. coli are provided in Supplementary information.

\section{Results}

\section{Training and Validation of a Machine Learning Model}

73 In this study, we train and test a model per organism with embeddings of prior known

74 transcriptional networks of the host cells to train three machine learning models for

75 combinatorial prediction of DEA from single inducers (Figure 1A). The best performing model

76 was selected using a validation dataset from two double-inducer conditions at two time points.

77 Experiments are then conducted with all remaining inducer combinations at two time points to

78 test the best performing model (in total 18 experimental conditions, 136 samples) (Figure 1B).

A
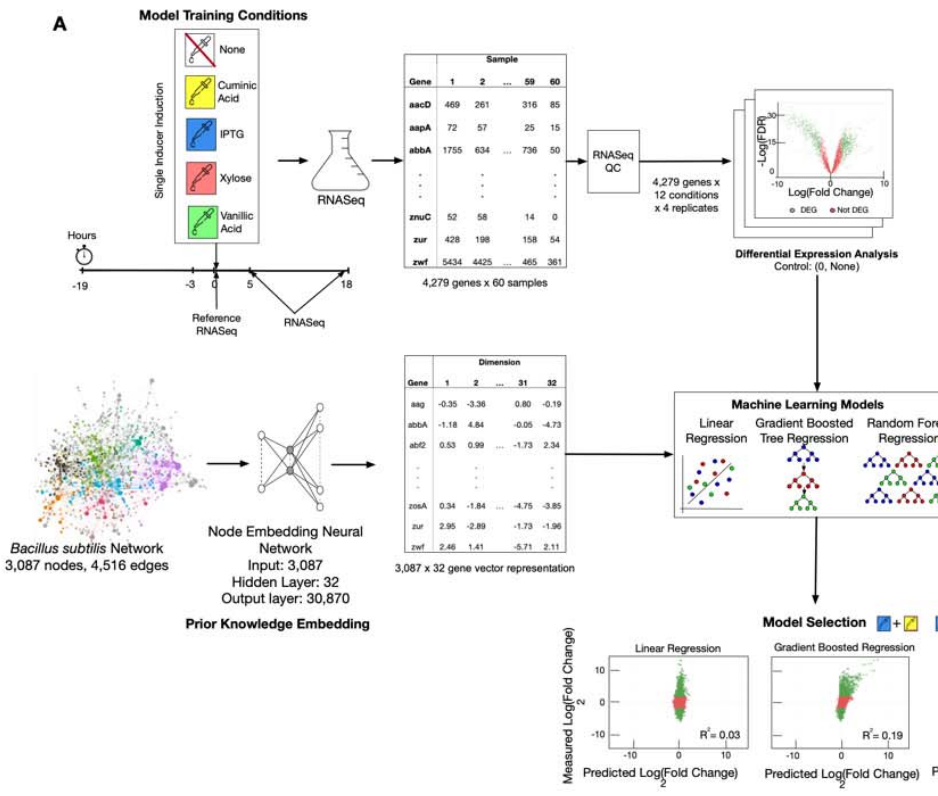

B

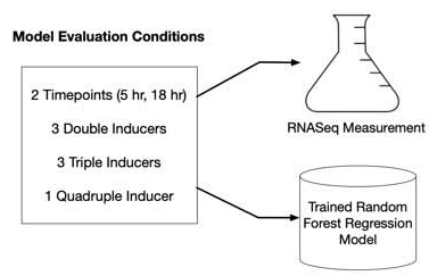

Figure 1: (A) Host response model experiment and knowledge integration for machine learning 
training and validation. For $B$. subtilis, RNASeq data was generated for single inducer conditions at each time point and passed through a configuration based differential expression analysis pipeline. Three machine learning models were validated with two held out pairs of inducer combinations. (B) The best model for the HRM is selected and tested with experiments run from all remaining combinations of inducers.

The HRM is formulated as a transcriptional dysregulation model trained with differential expression data and prior knowledge of gene networks of the host. The full set of conditions and

81 samples collected for the training, validation, and test sets can be found in Supplementary Table

821 and a detailed description of the model can be found in the Methods section.

84 Training data for E. coli included used EColiNet ${ }^{22}$ as the prior gene network and experimental

85 data that consisted of two inducers, Isopropyl $\beta$-d-1-thiogalactopyranoside (IPTG) and arabinose

86 at four time points $(5,6.5,8,18$ hours $)$. The test set was made up of the combination of the two

87 inducers at all four time points. The non-induced, earliest time point (5 hours) was used as a

88 control condition to measure host response using DEA for both training and testing data

89 (Supplementary Figure 1). Training data for B. subtilis consisted of a transcriptional regulatory

90 network $^{23}$ with experiments at two phases of growth (log and stationary), and four inducers:

91 IPTG, cuminic acid (CA), vanillic acid (VA), and xylose. The data was passed through a QC and

92 DEA pipeline which removed low-quality samples and genes (Supplementary Table 2).

94 A challenge faced by the HRM is that the number of differential expression comparisons can

95 have many factors, and thus, many design formulas. A Python-based configurable toolkit, which

96 we call omics_tools, that parallelizes the execution of DEA for the large condition space was 
97 developed to address this challenge. The tool aggregates the outputs from the parallelized runs

98 and combines all the data into a single unified dataset where each row represents a gene, it's

99 differential expression, statistical significance, and the condition for downstream machine

100 learning. Omics_tools uses edgeR ${ }^{24}$ to conduct DEA across the set of design variables. A control

101 condition of non-induced at the earliest time point was used to quantify the impact of induction

102 and time. The same control condition was measured for all runs of the experiment that made up

103 the training, validation and test set of data. The training corpus was formatted as follows: rows

104 represented genes in each experimental condition, while columns consisted of features of the

105 condition space, the node embedding features for the gene, and finally, the log fold change and

106 associated statistical significance of the gene in the condition as compared to the control. The

107 data can be found with the tutorial.

108

109 We find, surprisingly, a subspace representation of the individual responses enables prediction of

110 response to combination of inputs. Qualitative performance of the model was measured as the

111 number of dysregulated genes whose direction (up/down) was predicted correctly. Quantitative

112 performance was measured with an $\mathrm{R}^{2}$ metric comparing predicted versus actual fold-changes on

113 a logarithmic scale.

\section{HRM Predicts Transcriptional Response for E. coli}

115 The first question to address was whether the set of differentially expressed genes had a large

116 overlap between the train and test set. If so, then the task of machine learning would likely be

117 trivial. We measured the Jaccard similarity between the set of genes of pairs of conditions to

118 estimate the overlap (Supplementary Figure 2). The overlap between the conditions has a median 
of 0.2 with a standard deviation of 0.25 , indicating a significant difference between the train and test differentially expressed genes (DEGs).

122 Three machine learning models were trained in two ways: using only genes present in the prior

123 gene network versus using the whole transcriptome. To measure the impact of prior knowledge

124 on the model, the best performing machine learning model with prior knowledge was selected

125 and trained without prior knowledge to be used as a control. The criterion to label a gene as a

126 DEG for E. Coli are genes with absolute $\log _{2}$ (Fold Change $)>1.1$ and an FDR of $<0.01$.

127 Qualitatively and quantitatively it was clear that machine learning could accomplish the task, but

128 the impact of prior knowledge was marginal for E. coli (Table 1).

Table 1: E. coli results of qualitative and quantitative predictions for three different models using

131 two training methods as compared to a control method for model selection.

\begin{tabular}{|c|c|c|c|c}
\hline \multirow{2}{*}{ Model Name } & Prior Networks & & \\
& Used? & Training Method & Qualitative & Quantitative \\
\hline Gradient Boosted Regression & Yes & Genes only in network & $58.39 \%$ & 0.104 \\
\hline Gradient Boosted Regression & Yes & Whole Transcriptome & $58.07 \%$ & 0.103 \\
\hline Linear Regression & Yes & Genes only in network & $51.85 \%$ & 0.007 \\
\hline Linear Regression & Yes & Whole Transcriptome & $51.73 \%$ & 0.006 \\
\hline Random Forest Regression & Yes & Genes only in network & $90.20 \%$ & 0.887 \\
\hline Random Forest Regression & Yes & Whole Transcriptome & $87.66 \%$ & 0.846 \\
\hline
\end{tabular}




\begin{tabular}{|l|l|l|l|c}
\hline Random Forest Regression & No & Control & $89.59 \%$ & 0.829 \\
\hline
\end{tabular}

\section{Quantifying Prior Knowledge Impact for B. subtilis}

134 Similar to the validation framework of E. coli, all single inducers and a subset of the double

135 inducer data was used to train/validate the model (Table 2). Prior knowledge has a profound

136 impact on the $B$. subtilis predictions showing that models trained and tested with genes only

137 present in the prior network achieves $>90 \%$ accuracy. Most interestingly, a model that does not

138 use any prior knowledge of the host network achieved an $\mathrm{R}^{2}=0.306$, while one that used prior

139 knowledge achieved $\mathrm{R}^{2}=0.708$, a $2.5 \mathrm{x}$ increase in performance.

140

141 Table 2: B. subtilis results of qualitative and quantitative predictions for three different models

142 using two training methods as compared to a control method for model selection.

\begin{tabular}{|c|c|c|c|c|}
\hline & Prior & & \\
Motworks & & & \\
\hline Used? & Training Method & Qualitative & Quantitative \\
\hline Regression & Yes & Genes only in network & $51.20 \%$ & 0.227 \\
\hline Gradient Boosted & Yes & Whole Transcriptome & $50.43 \%$ & 0.194 \\
\hline Regression & Yes & Genes only in network & $47.24 \%$ & 0.031 \\
\hline Linear Regression & & & & \\
\hline
\end{tabular}




\begin{tabular}{|c|c|c|c|c|}
\multicolumn{1}{|c|}{ Linear Regression } & Yes & Whole Transcriptome & $47.27 \%$ & 0.029 \\
\hline Random Forest Regression & Yes & Genes only in network & $90.42 \%$ & 0.917 \\
\hline Random Forest Regression & Yes & Whole Transcriptome & $78.52 \%$ & 0.708 \\
\hline Random Forest Regression & No & Control & $53.04 \%$ & 0.306 \\
\hline
\end{tabular}

144 The discrepancy with E. coli can be explained by the heterogeneity of transcriptional response to

145 the larger set of induction conditions. We computed a rank, or Spearman, correlation between the

146 train and test induction conditions across all time points for both organisms. Specifically, this

147 would be three comparisons for E. coli (double induced to none and single induced) and 39

148 comparisons for B. subtilis (single induced to all combinations). A distribution of the statistic is

149 shown in Supplementary Figure 3. The larger distribution observed in the conditions of $B$.

150 subtilis versus E. coli explains the impact of prior knowledge.

\section{Testing the HRM with All Inducer Combinations in B. subtilis}

152 The best performing machine learning model, the random forest regressor, for the whole

153 transcriptome was selected to test all remaining combinations. Specifically, predictions at

154 remaining double, triple, and quadruple inducer conditions at the two time points were both

155 qualitatively and quantitatively evaluated (Figure 2). Certain conditions could not be evaluated

156 because there were less than two replicates that passed quality control (QC) (Supplementary

157 Table 2 and Discussion for more details). 


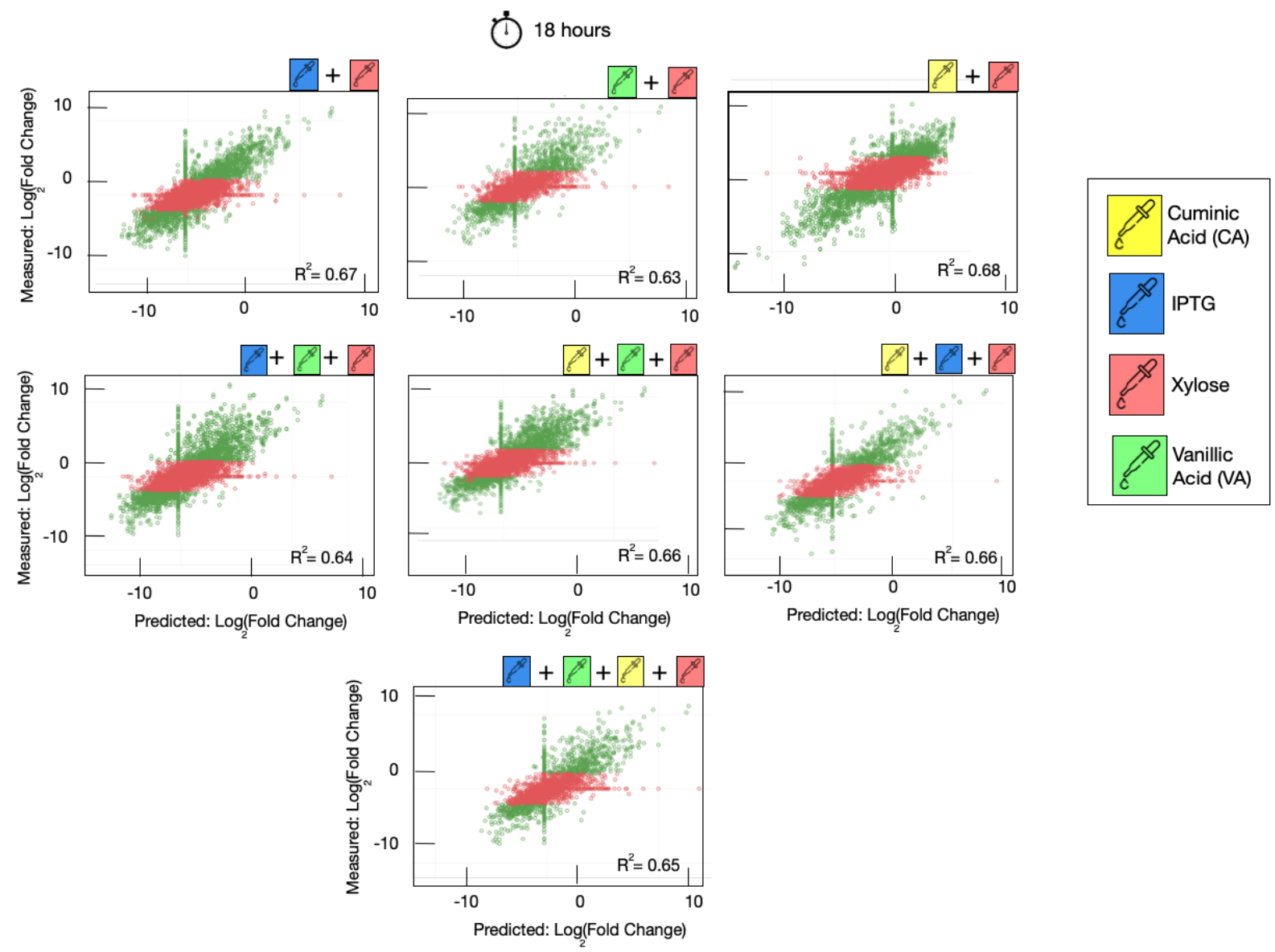

Figure 2: Predictions of transcriptional response -- $\log _{2}$ (Fold change) -- for B. subtilis at 18 hours for over 2,000 genes. Conditions tested do not overlap with training and validation sets.

Missing conditions are due to sample quality control. Red points are genes that are not differentially expressed while green genes are ones that are differentially expressed.

158 The model always maintained its performance within statistical error as the number of inducer

159 combinations increased (Figure 3A). Stationary phase predictions showed less variability and

160 achieved $>90 \%$ accuracy. The cells in log phase were of lower quality exhibiting lower OD and

161 RNA integrity (Supplementary Figure 4) across all inducer conditions. 
A
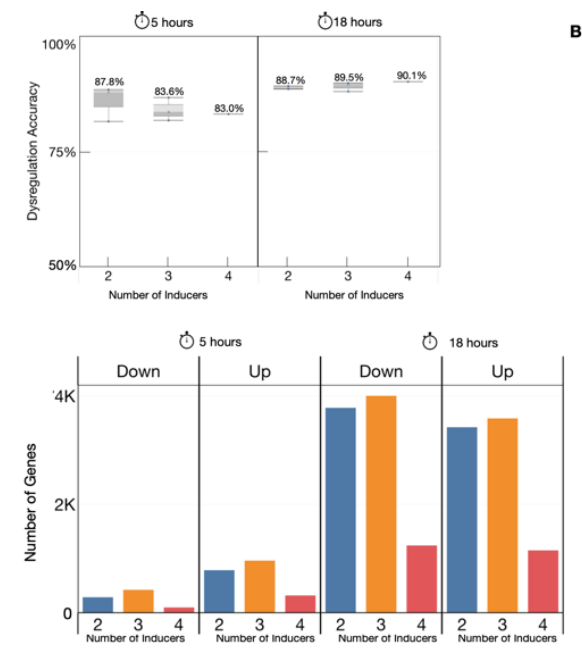
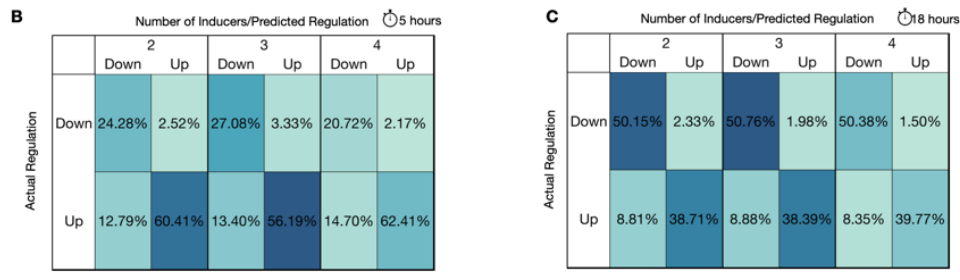

E

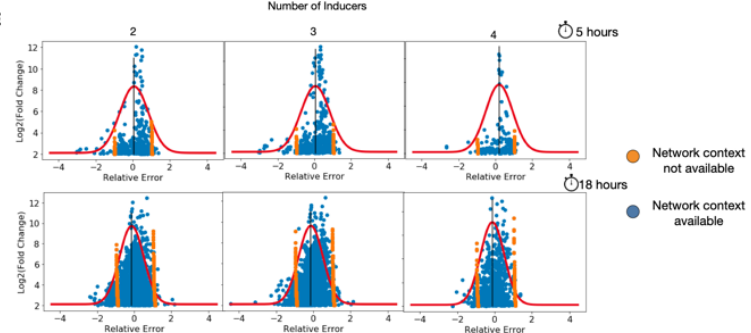

164 Figure 3: (A) Qualitative predictive performance of predictions on B. subtilis test set as it varies by the number of inducers. The performance stays within statistical error as the number of inducers increase. (B-C) Confusion matrices at 5 and 18 hours post-induction indicate that the model predicts more down-regulated genes as up-regulated. (D) The number of up and down regulated genes across inducers and time-points show 2x more up-regulated genes than downregulated genes at 5 hours which indicates a large class imbalance. The distribution is more evenly balanced at 18 hours. (E) Quantitative error analysis shows most of the errors occur at

$171 \mid \log _{2}$ (Fold change) $\mid<2.1$ or when the gene is not present in the network. As a matter of fact, the gene's not present in the network place an upper bound on the performance of the model.

174 Confusion matrices to assess the qualitative predictions show that the model had a more difficult 175 time predicting up-regulated genes at both time points (Figure 3B, 3C). Namely, there are more 176 up-regulated genes predicted as down-regulated than there are down-regulated predicted as up177 regulated. We should note that the log phase of growth had double the number of up-regulated 178 genes as down-regulated ones (Figure 3D), commonly known as a class imbalance. This provides an opportunity to get more up-regulated genes incorrect at that phase. When the class is 
180 balanced, as in the stationary phase, the model's predictions improve but hit an upper bound

181 which will be explained in the quantitative assessment.

182 Quantitatively, the relative error is normally distributed with larger error at smaller

183 dysregulations as those changes are harder to detect and predict. Genes with no network context

184 available were mapped to a single point in the embedding space and so the model always

185 predicted a constant value for all those genes (Figure 3E). The network is composed of 2,608

186 genes from the total 4,266 genes in our reference strain. While this is $>50 \%$ of the genome, genes

187 with no network information make up only $25 \%$ of the DEGs. Even so, these genes make up the

188 majority of qualitatively incorrect predictions and have greater than a single fold error (Table 3).

189 This results in an upper bound on the performance of a model as the majority of the model's

190 errors are due to a lack of network context for certain genes.

192 Table 3: Number of incorrect predictions with greater than a single fold change error are

193 primarily made up of genes with no embedding information.

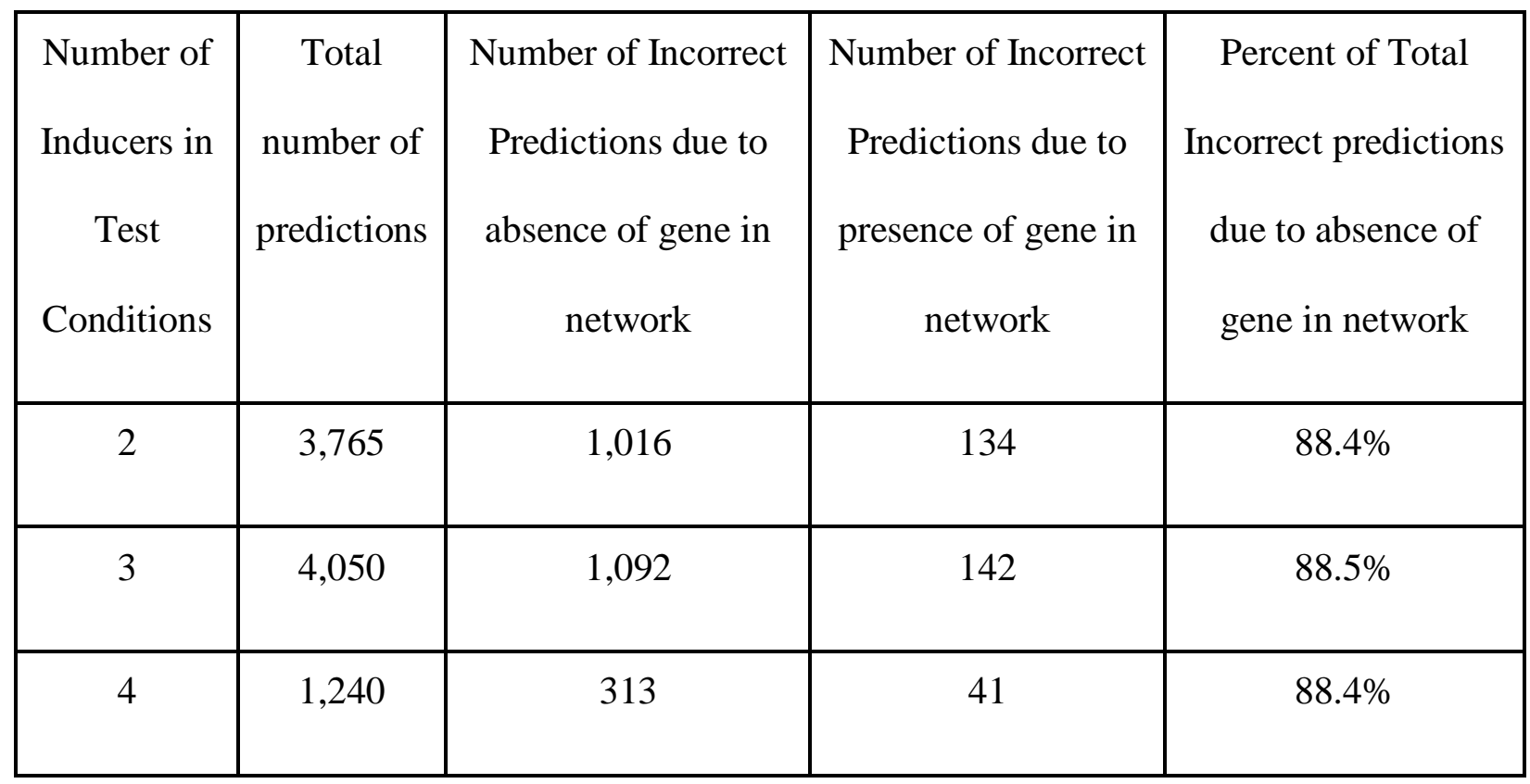




\section{Discussion}

196 In this paper, we present a machine learning model enriched with features from prior, known

197 transcriptional networks to qualitatively and quantitatively predict dysregulation of genes to a

198 combination of induction conditions at two phases of growth. We showed that the use of a prior

199 host network adds useful information to a model for it to make predictions of gene dysregulation

200 for unseen combinations of induction conditions.

202 A natural next question one would have is how our predictions would impact analyses

203 downstream of DEA, like enrichment analyses. These analyses help researchers gain mechanistic

204 insight into gene lists generated from $\mathrm{DEA}^{30}$. The goal here is to see how different the

205 mechanistic insights would be if a researcher uses the HRM's predictions from what they would

206 have observed if they executed the experiments. To answer this question, we added annotations

207 to the $B$. subtilis genes using SubtiWiki ${ }^{31}$ to conduct enrichment analysis of predicted versus

208 observed DEGs. No gene cluster file was publicly available to use standard enrichment tools. We

209 created a gene cluster file using data from SubtiWiki (Supplementary Data 2) and used gene set

210 enrichment analysis ${ }^{32,33}$ to identify up and down regulated pathways for each condition (Figure

211 4A). We evaluate the predictions by measuring the number of False Negatives (pathways that are

212 down regulated, but we predict it is not down regulated), False Positives (pathways that are not

213 down regulated, but we predict are down regulated), True Negatives (pathways that are not down

214 regulated and we do not predict them to be down regulated), and, finally, True Positives

215 (pathways that are downregulated and we predict them to be down regulated) (Figure 4B). The

216 same assessment is made for each test condition on the upregulated pathways (Figure 4C). As

217 one would expect, the inducers do not regulate many pathways and the model correctly identifies 
218 most of those pathways (gray boxes). Every row is a level 2 category in SubtiWiki that is

219 composed of multiple pathways. The number in each box represents the number of pathways in

220 the category.

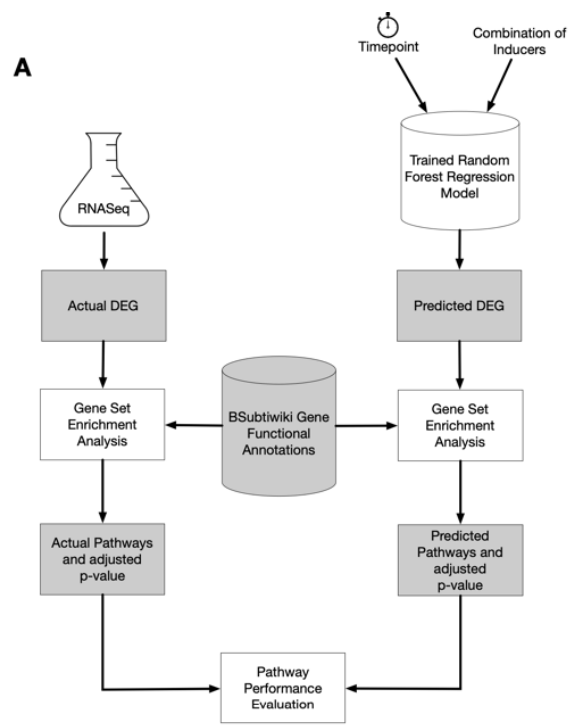

B

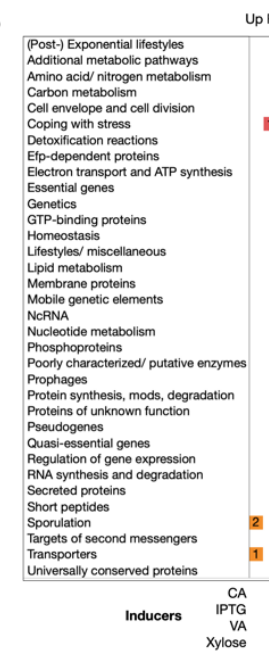

Up Regulated Pathways

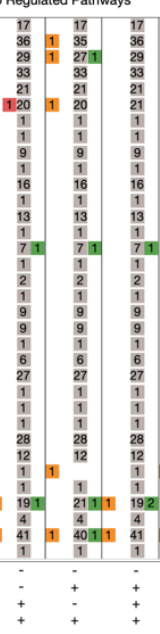

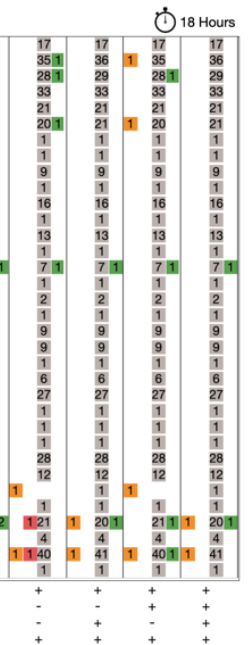

C

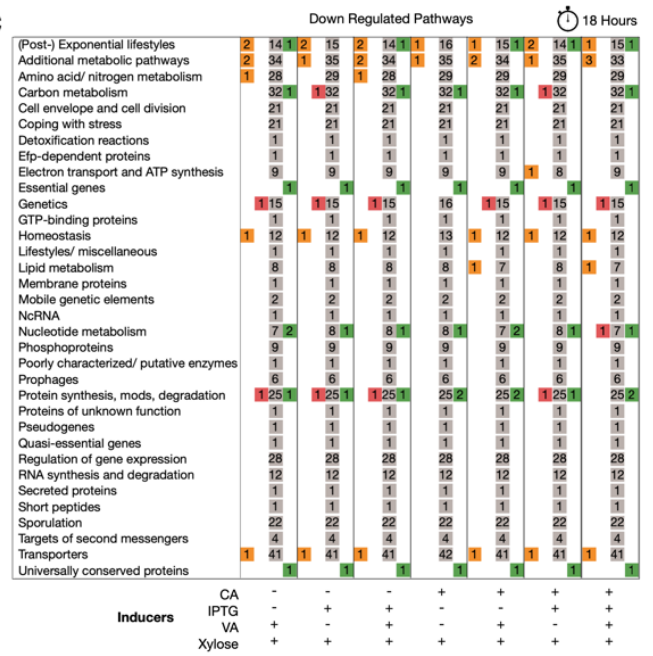

223 Figure 4: (A) Pathway analysis comparison of actual versus predicted set of DEGs using a gene

224 cluster file derived from SubtiWiki. (B-C) Level 2 categories of annotations in the database

225 composed of multiple down and up regulated pathways. The numbers inside each colored box

226 indicates the number of pathways that were FP, FN, TP, TN. As expected, the inducers do not 
229 Each pathway consists of a set of genes. Pathway analysis uses a statistical test (like a Fisher's

230 exact test) to identify dysregulated pathways by comparing the list of DEGs to each pathway's

231 gene set. Thus, we should also check the precision of identifying the regulation of a pathway.

232 This amounts to seeing how the predicted DEGs compare to the observed DEGs to identify if a

233 pathway is up or down regulated. We picked a False Positive (FP), False Negative (FN), and

234 True Positive (TP) sporulation pathway to assess the precision of the predictions (Figure 5).

235 The FP was selected from the CA+xylose condition, while the FN and TP were selected from the

236 IPTG+CA+VA+xylose condition, all at 18 hours. The genes that are in the bottom left quadrant

237 of each plot are the ones that contribute to the pathway's down-regulation status in both

238 predicted and actual settings. The genes in the top left quadrant contribute to the down-regulation

239 status of the pathway for predicted models but not in the observations. Finally, the genes in the

240 bottom right quadrant contribute to the pathway's down-regulation from the observations but not

241 from the predicted model. The vertical set of orange genes are ones that are not present in the

242 network and so the model predicts a constant for those expression values. The horizontal set of

243 green genes are genes that did not pass QC and thus could not be validated. As indicated in the

244 results section, these genes make up the majority of the genes that can be attributed to the

245 enrichment errors. It is clear from this analysis that future work should consider jointly

246 optimizing for differential expression as well as pathway inclusion. 

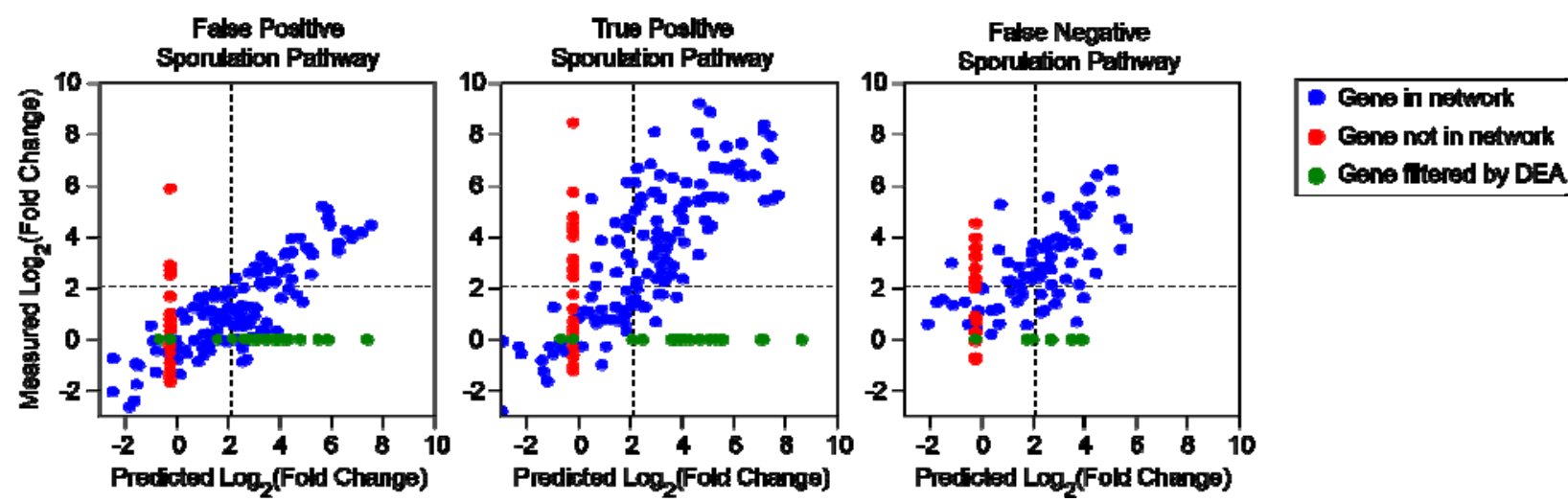

Figure 5: Predicted versus actual $\log _{2}$ (Fold change) of three pathways within the sporulation

\section{Two Stage Learning Model to Predict Differential Expression}

The goal to predict transcriptional response in a combinatorically large condition space from single conditions makes an end-to-end learning model with many free parameters underspecified and prone to generalizability errors ${ }^{25}$. To address this issue, we instead used a two stage learning

261 process:

262 1. Node embedding of Prior Knowledge: We applied the node2vec algorithm to derive vector features from the network that could be used in the downstream learning $\operatorname{task}^{26}$. 
node2 vec was selected because it is an unsupervised learning technique that balances depth and breadth first searches using a random walk to preserve both local and global length of the walks and the number of walks one takes from each node. A skip-gram model is then used to generate the vector embeddings in $\mathrm{R}^{\mathrm{N}}$, in our case $\mathrm{N}$ was 32 . We chose $\mathrm{N}=32$ after an assessment of model predictions on the train/validation set sweeping $\mathrm{N}$ between 8 , 16,32 , and 64 . The performance was not statistically different and so we chose a parameter that was large enough to provide the model with degrees of freedom to generalize but small enough to ensure the model does not overfit. We should note that the network need not be subtilis was $2608 / 4266$, which is $>50 \%$ for each organism. Genes that were not present in the network were mapped to the origin in $R^{32}$.

2. Machine Learning Models: We trained three machine learning models, a gradient boosted regressor, a linear regressor, and a random forest regressor, for their ability to predict the differential expression of a gene given the conditions of measurement and the derived network features. The models were trained on a regression task to minimize the error between predicted differential expression and the observed differential expression for a host's response to single inducers. The induction conditions were one hot encoded to enable the representation of multiple induction conditions. Since there were so few timepoints for E. coli and B. subtilis, it was not treated as a continuous variable and was also one hot encoded. 
287 The output predictions were evaluated using an $\mathrm{R}^{2}$ metric comparing predicted to actual

288 differential expression in $\log _{2}$ (Fold Change). We should note that only genes that were

289 differentially expressed were used to measure $\mathrm{R}^{2}$ as those are the ones most significant in

290 differential expression analysis. For $E$. coli, we defined a gene to be differentially expressed if it

291 had an absolute magnitude of $\log _{2}$ (Fold Change) $>1.1$ and FDR of $<0.05$, while for $B$. subtilis

292 We defined a gene to be differentially expressed if it had an absolute magnitude of $\log _{2}$ (Fold

293 Change) $>2.1$ and FDR of $<0.05$.

\section{Sample Preparation and Processing}

295 Wild type strains for B. subtilis (Bacillus Subtilis 168 Marburg) and E coli (E. coli K-12

296 MG1655) were cultured in M9 media consisting of 1X M9 media salts, 0.1mM CaCl2, $1 \mathrm{X}$ Trace

297 Salts, $1 \mathrm{mM}$ MgSO4, 0.05mM FeCl3/0.1mM C6H8O7, 0.2\% Casamino Acids, and 0.4\%

298 Glucose. The inducers used in this study were isopropyl $\beta$-D-1-thiogalactopyranoside $(0.001$

$299 \mathrm{M})$, arabinose $(25 \mathrm{mM})$, vanillic acid (0.001 M), cuminic acid (0.0001 M), and xylose (1\%).

301 Glycerol stocks were inoculated into M9 media in shake flasks, and the culture was grown

302 overnight for $18 \mathrm{~h}$ at $30 \square^{\circ} \mathrm{C}$ and $1000 \mathrm{rpm}$. The following day, cultures were diluted to OD 0.1 in

303 fresh M9 media and grown in 96-well plates under the same conditions for $3 \mathrm{~h}$. For induction,

304 cells were diluted a second time to OD 0.05 in the presence of inducers. Plates were incubated at

$30530 \square^{\circ} \mathrm{C}$ and $1000 \mathrm{rpm}$ for $5 \mathrm{~h}$ and $18 \mathrm{~h}$ and cultured cells were harvested and fixed with either

306 RNA protect (for E. coli) or methanol (B. subtilis). 
Total RNA was extracted using Magjet RNA extraction kit (Thermo) according to

309 manufacturer's instructions. RNA quality was assessed using Tapestation (Agilent). KAPA RNA

310 Hyperprep kit (Roche) was used for ribosomal RNA depletion and Illumina compatible library

311 preparation. Prepared library was loaded on a Illumina sequencer to generate 150bp paired end

312 reads.

314 Raw RNA-seq data was trimmed and quality filtered with trimmomatic (v0.36), reads were

315 aligned with bwa (v0.7.17). After alignment with bwa, the resulting sam files were sorted by

316 PICARD tools (v2.18.15) function SortSam, and then AddOrReplaceGroups is run on the sorted

317 sam. Gene-level quantification of counts was performed using the featureCounts function of

318 Rsubread (v1.34.4).

\section{Samples and Transcript Quality Control for B. subtilis}

320 A measure most often used to qualitatively and quantitatively assess a transcript's response is its

321 dysregulation as compared to a control. Differential expression analysis (DEA) is a standard

322 bioinformatics technique that measures this response to perturbations as compared to a control

323 condition ${ }^{19}$. DEA conducts custom normalization, dispersion modeling, and Bayesian

324 optimization to account for biological and experimental variability that is present in

325 transcriptional counts data to quantify the transcriptional response to a perturbation and measure

326 its statistical significance. While this method overcomes generalization errors that can arise from

327 artifacts of normalization of counts data across experiments, it performs strict quality control

328 (QC) at both the sample and gene level. These are listed below: 
1. Sample $\mathrm{QC}=$ The significance tests to reject the null hypothesis of the differentially expressed genes require $>2$ samples per condition. If this criterion is not met then DEA cannot be conducted.

2. Gene $\mathrm{QC}=\mathrm{DEA}$ tools fits the Cox-Reid profile-adjusted dispersion to a set of normalized expressions across conditions. Genes that do not fit this profile are labeled as outliers and removed from DEA.

335 Three Boolean metrics were used to measure sample quality:

336 1. Number of mapped reads $\geq 500 \mathrm{~K}$

337 2. Count of all annotated genes $\geq 500 \mathrm{~K}$

338 3. Replicate correlation of a condition $>0.9$

340 If any of these metrics did not pass, the sample would be flagged as a low quality sample and not

341 used for downstream analysis. For the B. subtilis experiments, we also collected OD600

342 measurements from a plate reader to correlate population with potential sample dropouts. We did

343 not find a clear, discriminative correlation between this measurement and sample dropout for a

344 condition, but the log phase measurements (timepoint 5.0) did have a lower OD on average and

345 had twice as many samples that did not pass QC than stationary phase samples (timepoint 18.0).

346 In conditions where only two replicates were available, differential expression analysis was not

347 conducted and so those conditions could not be validated (Supplementary Table 2). All single

348 inducer conditions for B. subtilis that passed QC were used to train the model. It should be noted,

349 though, that if a sample passed QC, that did not mean all genes in that sample passed edgeR's

350 outlier detection method. edgeR fits normalized counts to a Cox-Ried dispersion model with a

351 Bayseian optimization algorithm. A gene is removed by edgeR if it does not fit this dispersion 
352 model. While one can pass in a custom dispersion model per condition, we chose to use edgeR's

353 default, as development of custom noise models across the condition space was out of scope of

354 this effort (Supplementary Table 3).

\section{Funding}

356 Any opinions, findings and conclusions or recommendations expressed in this material are those of the

357 author(s) and do not necessarily reflect the views of the Defense Advanced Research Projects Agency

358 (DARPA), the Department of Defense, or the United States Government. This material is based upon

359 work supported by the Defense Advanced Research Projects Agency (DARPA) and the Air Force

360 Research Laboratory under Contract No. FA8750-17-C-0231 (and related contracts by SD2 Publication

361 Consortium Members).

\section{Data and Code Availability}

363 The manuscript is accompanied with three code repositories that are fully documented with

364 example python notebooks. The data for the publication is placed with the tutorials to ensure

365 reproducibility of results.

366 1. A repository that includes the capability to train, validate, and test a machine learning

367 model in a combinatorically large condition space. https://github.com/sd2e/CDM

368 2. A repository that includes a scaled, configurable differential expression analysis pipeline:

369 https://github.com/SD2E/omics_tools.

370 3. A test-harness for machine learning models to make apples-to-apples comparisons of

371 training and testing models: https://github.com/SD2E/test-harness 


\section{Author Contributions}

373 M.E., A. E. B, H. E., and E. Y. led the analysis plan, analysis, and design of experiments for E.

374 coli and B. subtilis. H.D. also contributed to B. subtilis experiments. D. B., C. B., P. M, K. C.,

375 performed the experiments. J. U., M. W., M. V., G. Z., N. G., J. F., and J. S. designed and

376 automated the data processing, quality control, and data/analysis collaboration infrastructure. M.

377 E., G. Z, and A. C. developed, scaled, and automated the execution the differential expression

378 analysis pipeline. M. E. and H. E. developed the machine learning test harness. M. E. and H. E.

379 developed the Host Response Model Library. C. V., B.G., J.S., and Y.D are PIs that managed the

380 program and provided technical guidance. M. E., A. E. B., and E. Y. prepared the manuscript.

\section{Competing Interests}

382 The authors report no competing interests. 


\section{References}

385

386

387

388

389

390

391

392

393

394

395

396

397

398

399

400

401

402

403

404

405

406

407

408

409

410

411

412

413

414

415

416

417

418

419

420

421

422

423

424

425

426

427

428

429

430

431

432

1. Helmann, J. D. et al. Global transcriptional response of Bacillus subtilis to heat shock. J. Bacteriol. 183, 7318-7328 (2001).

2. Gao, H. et al. Global transcriptome analysis of the heat shock response of Shewanella oneidensis. J. Bacteriol. 186, 7796-7803 (2004).

3. Hengge-Aronis, R. Back to log phase: sigma $S$ as a global regulator in the osmotic control of gene expression in Escherichia coli. Mol. Microbiol. 21, 887-893 (1996).

4. Soufi, B. et al. Global analysis of the yeast osmotic stress response by quantitative proteomics. Mol. Biosyst. 5, 1337-1346 (2009).

5. Erickson, D. W. et al. A global resource allocation strategy governs growth transition kinetics of Escherichia coli. Nature 551, 119-123 (2017).

6. Alexander, H., Rouco, M., Haley, S. T. \& Dyhrman, S. T. Transcriptional response of Emiliania huxleyi under changing nutrient environments in the North Pacific Subtropical Gyre. Environ. Microbiol. 22, 1847-1860 (2020).

7. Dunn, T. M., Hahn, S., Ogden, S. \& Schleif, R. F. An operator at -280 base pairs that is required for repression of araBAD operon promoter: addition of DNA helical turns between the operator and promoter cyclically hinders repression. Proc. Natl. Acad. Sci. USA 81, 5017-5020 (1984).

8. Harmer, T., Wu, M. \& Schleif, R. The role of rigidity in DNA looping-unlooping by AraC. Proc. Natl. Acad. Sci. USA 98, 427-431 (2001).

9. Martin, K., Huo, L. \& Schleif, R. F. The DNA loop model for ara repression: AraC protein occupies the proposed loop sites in vivo and repression-negative mutations lie in these same sites. Proc. Natl. Acad. Sci. USA 83, 3654-3658 (1986).

10. Brunton, S. L., Proctor, J. L. \& Kutz, J. N. Discovering governing equations from data by sparse identification of nonlinear dynamical systems. Proc. Natl. Acad. Sci. USA 113, 3932-3937 (2016).

11. Champion, K., Lusch, B., Kutz, J. N. \& Brunton, S. L. Data-driven discovery of coordinates and governing equations. Proc. Natl. Acad. Sci. USA 116, 22445-22451 (2019).

12. Adam, G. et al. Machine learning approaches to drug response prediction: challenges and recent progress. NPJ Precis. Oncol. 4, 19 (2020).

13. Fitzgerald, J. B., Schoeberl, B., Nielsen, U. B. \& Sorger, P. K. Systems biology and combination therapy in the quest for clinical efficacy. Nat. Chem. Biol. 2, 458-466 (2006).

14. Kuru, H. I., Tastan, O. \& Cicek, A. E. Matchmaker: A deep learning framework for drug synergy prediction. BioRxiv (2020). doi:10.1101/2020.05.24.113241

15. Li, J., Tong, X.-Y., Zhu, L.-D. \& Zhang, H.-Y. A machine learning method for drug combination prediction. Front. Genet. 11, 1000 (2020).

16. Chen, G., Tsoi, A., Xu, H. \& Zheng, W. J. Predict effective drug combination by deep belief network and ontology fingerprints. J. Biomed. Inform. 85, 149-154 (2018).

17. Xue, Y., Ding, M. Q. \& Lu, X. Learning to encode cellular responses to systematic perturbations with deep generative models. NPJ Syst. Biol. Appl. 6, 35 (2020).

18. Costa-Silva, J., Domingues, D. \& Lopes, F. M. RNA-Seq differential expression analysis: An extended review and a software tool. PLoS One 12, e0190152 (2017).

19. Schmauch, B. et al. A deep learning model to predict RNA-Seq expression of tumours from whole slide images. Nat. Commun. 11, 3877 (2020).

20. Schmidt, F., Kern, F. \& Schulz, M. H. Integrative prediction of gene expression with chromatin accessibility and conformation data. Epigenetics Chromatin 13, 4 (2020).

21. Abbas-Aghababazadeh, F., Li, Q. \& Fridley, B. L. Comparison of normalization approaches for gene expression studies completed with high-throughput sequencing. PLoS One 13, 
e0206312 (2018).

22. Kim, H., Shim, J. E., Shin, J. \& Lee, I. EcoliNet: a database of cofunctional gene network for Escherichia coli. Database (Oxford) 2015, (2015).

23. Arrieta-Ortiz, M. L. et al. An experimentally supported model of the Bacillus subtilis global transcriptional regulatory network. Mol. Syst. Biol. 11, 839 (2015).

24. Robinson, M. D., McCarthy, D. J. \& Smyth, G. K. edgeR: a Bioconductor package for differential expression analysis of digital gene expression data. Bioinformatics 26, 139-140 (2010).

25. D'Amour, A. et al. Underspecification Presents Challenges for Credibility in Modern Machine Learning. arXiv (2020).

26. Grover, A. \& Leskovec, J. node2vec: Scalable Feature Learning for Networks. KDD 2016, 855-864 (2016).

27. Kim, M., Baek, S. H. \& Song, M. Relation extraction for biological pathway construction using node2vec. BMC Bioinformatics 19, 206 (2018).

28. Ata, S. K. et al. Integrating node embeddings and biological annotations for genes to predict disease-gene associations. BMC Syst. Biol. 12, 138 (2018).

29. Nelson, W. et al. To embed or not: network embedding as a paradigm in computational biology. Front. Genet. 10, 381 (2019).

30. Reimand, J. et al. Pathway enrichment analysis and visualization of omics data using g:Profiler, GSEA, Cytoscape and EnrichmentMap. Nat. Protoc. 14, 482-517 (2019).

31. Zhu, B. \& Stülke, J. SubtiWiki in 2018: from genes and proteins to functional network annotation of the model organism Bacillus subtilis. Nucleic Acids Res. 46, D743-D748 (2018).

32. Chen, E. Y. et al. Enrichr: interactive and collaborative HTML5 gene list enrichment analysis tool. BMC Bioinformatics 14, 128 (2013).

33. Kuleshov, M. V. et al. Enrichr: a comprehensive gene set enrichment analysis web server 2016 update. Nucleic Acids Res. 44, W90-7 (2016). 


\section{A Model Training Conditions}
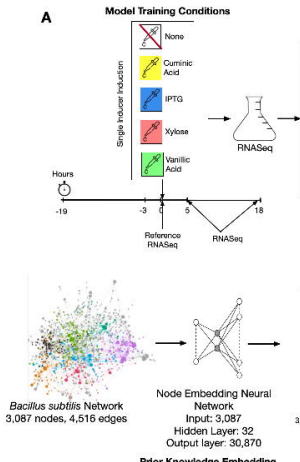

Prior Knowledge Embedding

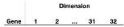

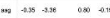

abea 1.11 \& 4 st un 4.73

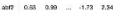

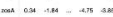

is $2: 2=20$

2ut $2.46 \quad 1.51 \quad 5 \% \quad 2.4$

$3,087 \times 32$ gene vector representrstion $\underset{00}{\text { Rnusur }}$

$4, \begin{aligned} & 1,279 \text { gures } x \\ & 12 \text { conditions }\end{aligned}$ 2. 4 roplizates

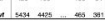

4,279 genes $x$ fo samales

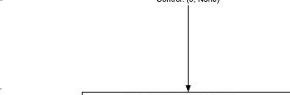

Machine Learning Models

Linear Gredient Ecosted Random Fores

Fegrassion Trea Regrassion Regression

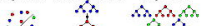

$\because \%:$

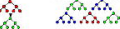
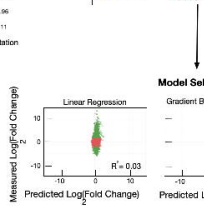

Model Selection +7 \& $\mid r-10,693$ print:

Graxant Bonstar Fapression
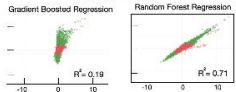

B

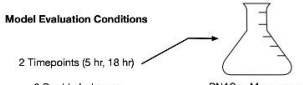

3 Double Inducers

RNASeq Measurement

3 Triple Inducers

1 Quscruple Inducer

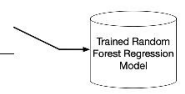


(1) 18 heurs

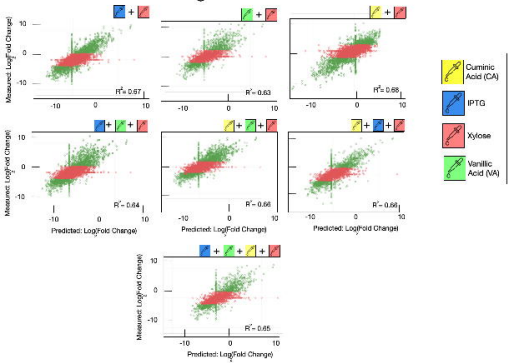



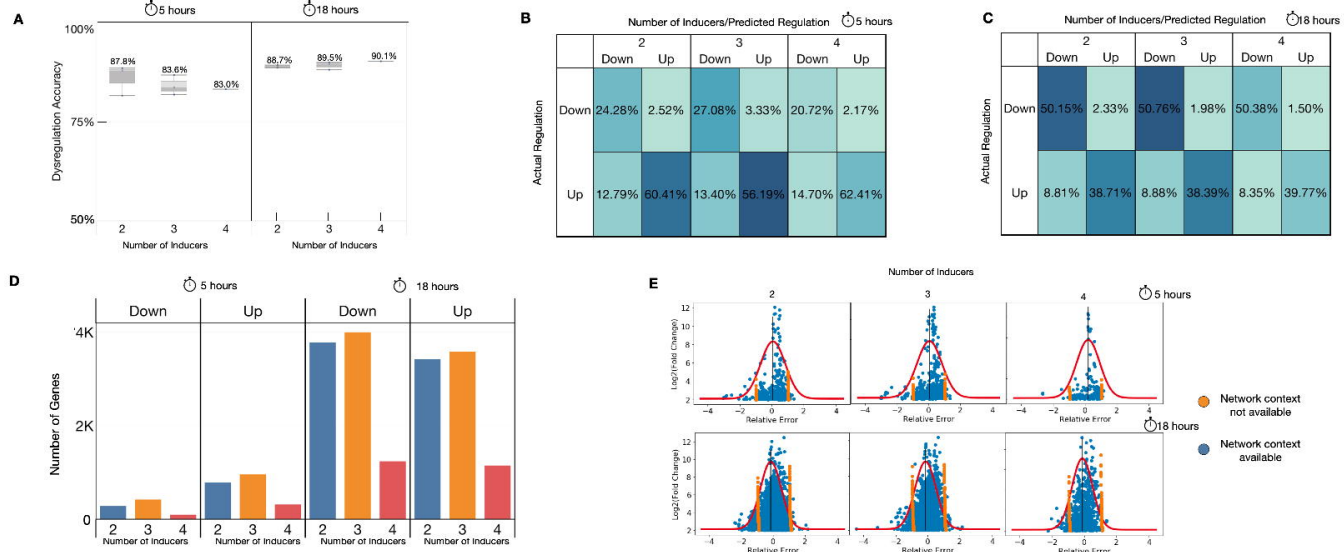
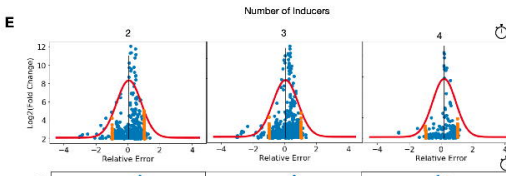

ல்s hours
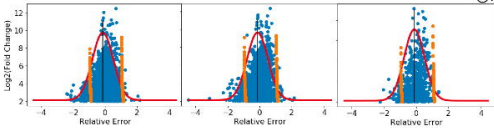

Network context not available

- Network context available 


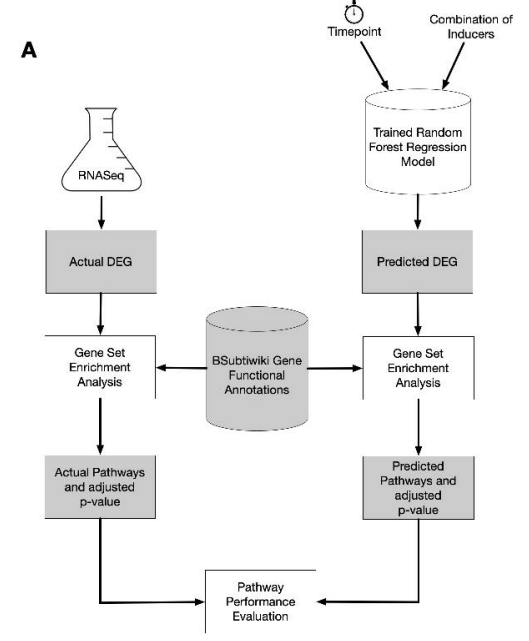

B

\begin{tabular}{|c|c|c|c|c|c|c|c|c|c|c|c|}
\hline & \\
\hline (Post-) Exponential Ifestyles & & 17 & & 17 & & 17 & & 17 & & 17 & 17 \\
\hline Additional metabolic pathways & & 36 & 1 & 35 & 36 & 351 & & 36 & 1 & $\frac{11}{35}$ & 36 \\
\hline Amino acid/ nitrogen metzbolism & & 29 & 1 & 271 & 29 & $2 B 1$ & & 29 & & 281 & 29 \\
\hline Carbon metabolism & & 33 & & 33 & 33 & 33 & & 33 & & 33 & 33 \\
\hline Cell envelope and cell division & & 21 & & 21 & 21 & 21 & & 21 & & 21 & 21 \\
\hline Coping with stress & & 120 & 1 & 20 & 21 & 201 & & 21 & 1 & 20 & 21 \\
\hline Detoxification reactions & & 1 & & 1 & 1 & 1 & & 1 & & 1 & 1 \\
\hline Eip-dependert proteins & & 1 & & 1 & 1 & 1 & & 11 & & 1 & 1 \\
\hline Electron transport and ATP synthesis & & 9 & & 9 & 9 & 9 & & 9 & & 9 & 9 \\
\hline Essential genes & & 1 & & 1 & 1 & 1 & & 1 & & 1 & 1 \\
\hline Genetics & & 16 & & 16 & 16 & 16 & & 16 & & 16 & 16 \\
\hline GTP-binding proteins & & 1 & & 1 & 1 & 1 & & 1 & & 1 & 1 \\
\hline Homeostasis & & $\frac{1}{13}$ & & $\frac{1}{13}$ & 13 & $\frac{1}{13}$ & & 13 & & 13 & $\frac{1}{13}$ \\
\hline Lifestyles/miscellaneous & & 1 & & 1 & 1 & 1 & & 1 & & 1 & 1 \\
\hline $\begin{array}{l}\text { Lioid metabolism } \\
\text { Lach }\end{array}$ & & 71 & & 71 & 71 & 71 & & 71 & & 71 & 711 \\
\hline Membrane proteins & & 1 & & 1 & 1 & 1 & & 1 & & 1 & 1 \\
\hline Mobile genetic elements & & 2 & & 2 & 2 & 2 & & 2 & & 2 & 2 \\
\hline NCRNA & & 1 & & $\frac{1}{1}$ & $\frac{1}{1}$ & $\frac{1}{1}$ & & $\frac{2}{1}$ & & $\frac{2}{1}$ & $\frac{1}{1}$ \\
\hline Nucleotide metabolism & & 9 & & 9 & 9 & 9 & & 9 & & 9 & 9 \\
\hline Phosphoproteins & & 9 & & 9 & 9 & 9 & & 9 & & 9 & 9 \\
\hline Poorly characterized/ putative enzymes & & 1 & & 1 & $\frac{2}{1}$ & 1 & & $\frac{\pi}{1}$ & & $\frac{0}{1}$ & 1 \\
\hline Prophages & & 6 & & 6 & 6 & 6 & & 6 & & 6 & 6 \\
\hline Protein synthesis, mods, degradation & & 27 & & 27 & 27 & 27 & & 27 & & 27 & 27 \\
\hline Proteins of unknown function & & 1 & & 1 & 1 & 1 & & 1 & & 1 & 1 \\
\hline Pseudogenes & & 1 & & 1 & 1 & 1 & & 1 & & 1 & 1 \\
\hline Quasi-essential genes & & 1 & & 1 & 1 & 1 & & 1 & & 1 & 1 \\
\hline Regulation of gene expression & & 28 & & $2 B$ & 28 & 28 & & 28 & & 28 & 28 \\
\hline RNA synthesis and degradation & & 12 & & 12 & 12 & 12 & & 12 & & 12 & 12 \\
\hline Secreted proteins & & 1 & 1 & & 1 & 1 & & 1 & 1 & & 1 \\
\hline Shart peptides & & 1 & & 1 & 1 & 1 & & 1 & & & 1 \\
\hline Sporulation & 2 & 191 & & 2111 & 192 & 121 & 1 & 201 & & 2111 & 201 \\
\hline Targets of second messengers & & 4 & & & 4 & 4 & & 4 & & 4 & 4 \\
\hline Transporters & 1 & $\frac{4}{41}$ & 1 & 4011 & $\frac{4}{41}$ & 1140 & 1 & 41 & 1 & 4011 & $\frac{4}{41}$ \\
\hline Universally consenved proteins & & 1 & & & 1 & 1 & & 1 & & 1 & 1 \\
\hline & & - & & - & - & + & & & & & \\
\hline IPTG & & . & & + & + & - & & - & & + & + \\
\hline IPIG & & + & & - & + & - & & + & & - & + \\
\hline Xylose & & + & & + & + & + & & + & & + & + \\
\hline
\end{tabular}

C

(Post-) Exponential lifestyles Additional metabolic pathways Amino acid nitrogen metabolism

Coping with stress

Detoxification reaction

Eto-desication reactions

Electron transport and ATP synthesis

Essential genes

Genetics

GTP-binding proteins

Homeostasis

Lipid metabolism

False Negative

False Positive

True Negative

True Positive Mobile genetic elements NCPNA

Nucleotide metabolism Phosphoproteins
Down Regulated Pathways

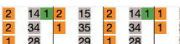
\begin{tabular}{|cc|r|}
1 & 28 & 29 \\
& 321 & 132 \\
\hline & & 21
\end{tabular} \begin{tabular}{l|r}
321 & -132 \\
21 & 21
\end{tabular} $\frac{21}{1} \quad \frac{21}{1}$ $\frac{1}{9}$ ${ }_{115^{1}}^{1}$ $1 \frac{1}{12} 1$ Poorly characterized/ putative enzymes Propheges Protein synthesis, mods, degradation Proteins of unknown function Pseucogenes Quasi-essential genes Regulation of gene expression Secroted protens Shected protens Sporuation

Targets of second messengers Universally conserved protein

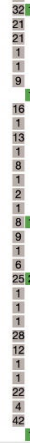

(1) 18 Hours

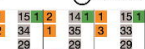

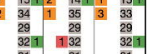

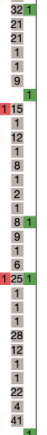

\begin{tabular}{l|l|l}
21 & 21 & 21 \\
21 & 21 & 21
\end{tabular} 


$$
\text { H }
$$

